\title{
A New Inner Vertex Detector for STAR
}

\author{
H. Wieman, ${ }^{\text {a }}$ F. Bieser, ${ }^{\text {a }}$ S. Kleinfelder, ${ }^{\text {a }}$ H.S. Matis, ${ }^{\text {a }}$ \\ P. Nevski, ${ }^{b}$ G. Rai, ${ }^{a}$ N. Smirnov ${ }^{c}$ and the STAR Collaboration \\ ${ }^{a}$ Lawrence Berkeley National Laboratory, Berkeley, CA 94720 \\ ${ }^{\mathrm{b}}$ Brookhaven National Laboratory, Upton, NY 11973 \\ ${ }^{\mathrm{c}}$ Yale University, New Haven, CT 06520
}

\begin{abstract}
We report on design considerations for a new inner vertex detector for the STAR experiment at RHIC. A brief description of the STAR experiment and motivation for a high resolution vertex detector are presented.
\end{abstract}

\section{Introduction}

There are compelling physics reasons to expand the capabilities of the STAR experiment at RHIC with a new high resolution inner vertex detector. This new detector, which is being designed to measure D mesons, will provide STAR with opportunity to measure open charm.

\section{STAR Experiment}

RHIC is a newly commissioned Relativistic Heavy Ion Collider located at Brookhaven National Laboratory. It will be able to collide $\mathrm{Au}+\mathrm{Au}$ ions at $\sqrt{s_{N N}}=200 \mathrm{GeV}$. The STAR experiment is located at one of the four instrumented intersection regions. This system is designed to cover a large solid angle and is currently composed of a large cylindrical TPC in a solenoidal magnet - the classic design for a collider geometry. There is a central trigger barrel array of plastic scintillators around the TPC cylinder. Additional detectors are in various stages of construction and installation. These include electromagnetic calorimeter, forward TPCs, the SVT (a 3 layer silicon drift chamber vertex detector [1]) and a 4th layer of silicon strip detectors. This 
summer (2000) RHIC, operating at $\sqrt{s_{N N}}=130 \mathrm{GeV}$, produced the first collisions of $\mathrm{Au}+\mathrm{Au}$. A typical image of reconstructed tracks measured in the TPC is shown in Figure 1. Next summer (2001) RHIC will run at full energy, $\sqrt{s_{N N}}=200 \mathrm{GeV}$.

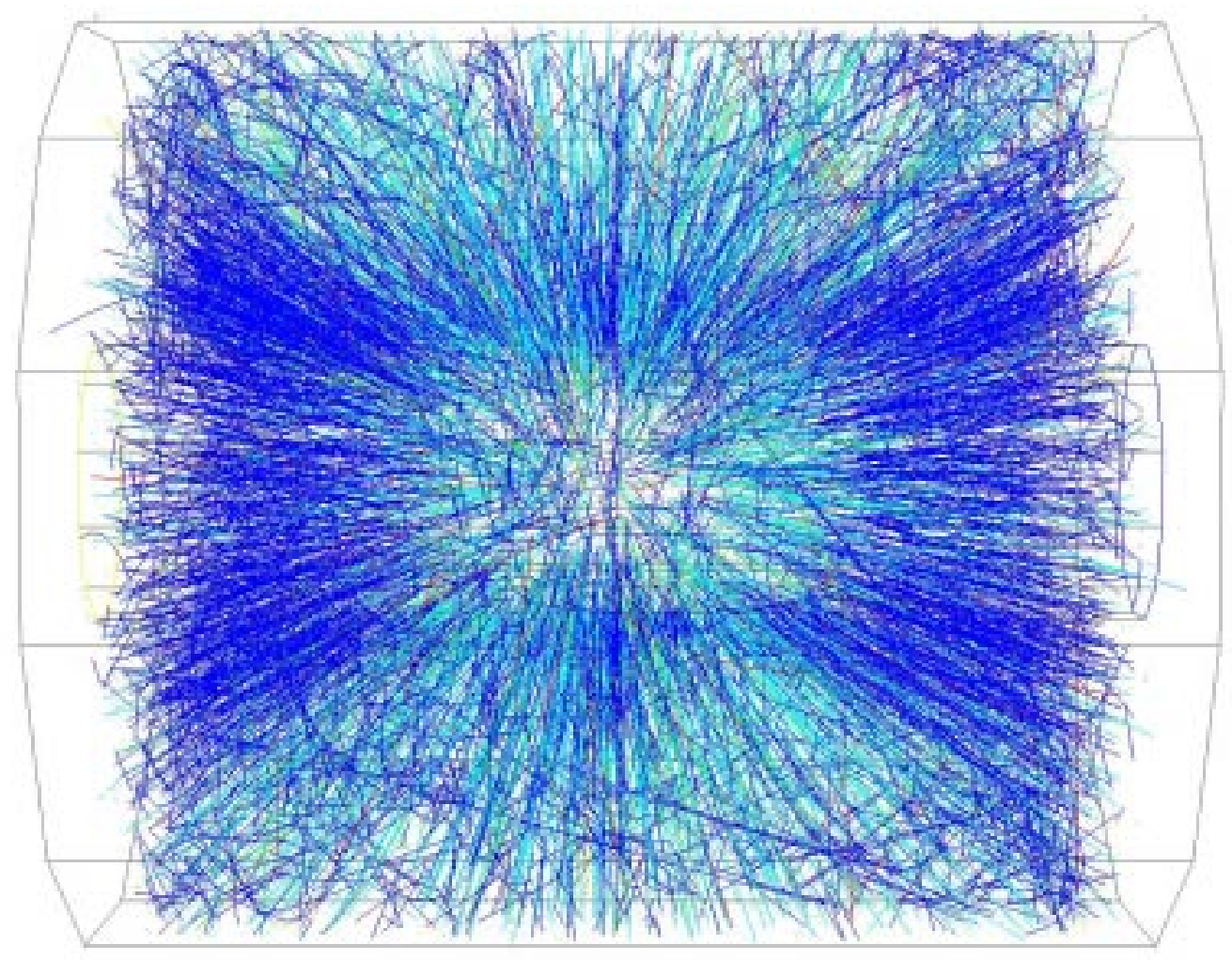

Fig. 1. Typical Reconstructed Central Au $+\mathrm{Au}$ Collision

\section{Motivation for an Inner Detector}

The primary motivations for studying nucleus-nucleus collisions at RHIC and the Large Hadron Collider (LHC) are to understand the behavior of bulk matter governed by QCD and to identify the Quark-Gluon Plasma (QGP). The nature of this deconfined matter and its subsequent conversion to hadronic matter are of fundamental importance.

The STAR detector at RHIC is equipped with excellent particle identification and tracking capabilities. Most of the baryons and mesons reconstructed in the TPC are created during the hadronization process. The STAR physics program is considerably enhanced by the detection of particles which convey information about the QGP epoch and are largely unaffected by hadronization. 
Charmed particles are one such type of particle. Their detection requires the development of a state of the art, high-resolution pixel detector.

Charmed quarks are heavy and consequently very difficult to produce in hadronic interactions. However, they are created in the hot partonic phase of the QGP. Thus, a measurement of D mesons (short-lived particles containing one charmed quark) reflects the in-situ charm content. The enhancement of D meson production can be studied with respect to suitable control parameters (e.g., beam energy and impact parameter).

\section{Feasibility of a D Detector}

As recent advances in silicon technology have been made, we have reexamined the question - is it possible to identify D mesons in heavy-ion collisions? The SLD experiment at SLAC produced an exceptional vertex detector[2] using $\mathrm{CCD}[3]$ technology. This detector, which had $4 \mu \mathrm{m}$ spatial resolution, successfully measured D mesons. The ALICE experiment is building an inner tracking system that will have D measuring capability.[4] Another effort by the LEPSI Strasbourg group has designed, fabricated and tested a novel detector structure $\left(64 \times 64,20 \times 20 \mu m^{2}\right.$ elements) based on an emerging technology called Active Pixel Sensors (APS) which uses a CMOS process[5],[6]. The CMOS APS pixels detect minimizing ionizing particles by collecting electrons left in the underlying epitaxial layer by the passage of the ionizing particle. The electrons diffuse from the field free epitaxial layer into $\mathrm{n}$ wells centered in each pixel. The epitaxial layer is sufficiently thick, 5 to $15 \mu \mathrm{m}$, so enough electrons are produced to give a good signal to noise ratio. Active elements on each pixel selectively switch the signal onto readout traces on the chip. The potential advantage of this technology is the ease with which supporting electronic functions such as zero suppression can be added to the detector chip. Both technologies allow the substrate to be thinned $(50 \mu \mathrm{m})$ which is a major consideration for successfully isolating D mesons.

The challenge of finding D's at RHIC is daunting, but not impossible with the use of a high resolution and thin detector. A typical Au on Au central collision yields thousands of tracks from the primary vertex. Detection of D's requires the isolation of the $\mathrm{D}$ decay vertex from all the primary tracks. The $\mathrm{D}_{0} \mathrm{c} \tau$ is $126 \mu \mathrm{m}$, while the $\mathrm{D}_{ \pm} \mathrm{c} \tau$ is $320 \mu \mathrm{m}$. In a heavy ion collider $\mathrm{D}$ mesons of interest have velocities, $\beta$, significantly less than unity and therefore decay closer to the vertex than their $\mathrm{c} \tau$ value. These microscopic decay lengths and the accompanying background set the requirements for a high-resolution vertex detector. A pixel detector with a feature size of $10-20 \mu \mathrm{m}$ is needed. Additionally, the detector must be made thin to minimize multiple scattering and in turn achieve a high pointing accuracy. With a thinned detector of this 
resolution, the beam pipe and the distance to the first layer become significant contributions to the resolution. The ability to isolate the primary vertex tracks from $\mathrm{D}$ candidate pions is illustrated in Figure 2 for several cases. For this simulation, the beam pipe is at $2.32 \mathrm{~cm}$ and the two silicon layers are at $2.80 \mathrm{~cm}$ and $3.82 \mathrm{~cm}$. This figure shows the integrated fraction of tracks that can be excluded from the invariant mass combinatorics by making an impact parameter cut. Taking the ultimate example with a $40 \mu \mathrm{m}$ thick Silicon detector and no beam pipe, an impact parameter cut of $50 \mu$ m leaves $10 \%$ of the primary tracks contributing to the background. Where we model the configuration used in the SLD experiment with more silicon, a beryllium backer and a beryllium beam pipe requires moving the cut to $140 \mu \mathrm{m}$ to achieve the same exclusion fraction.

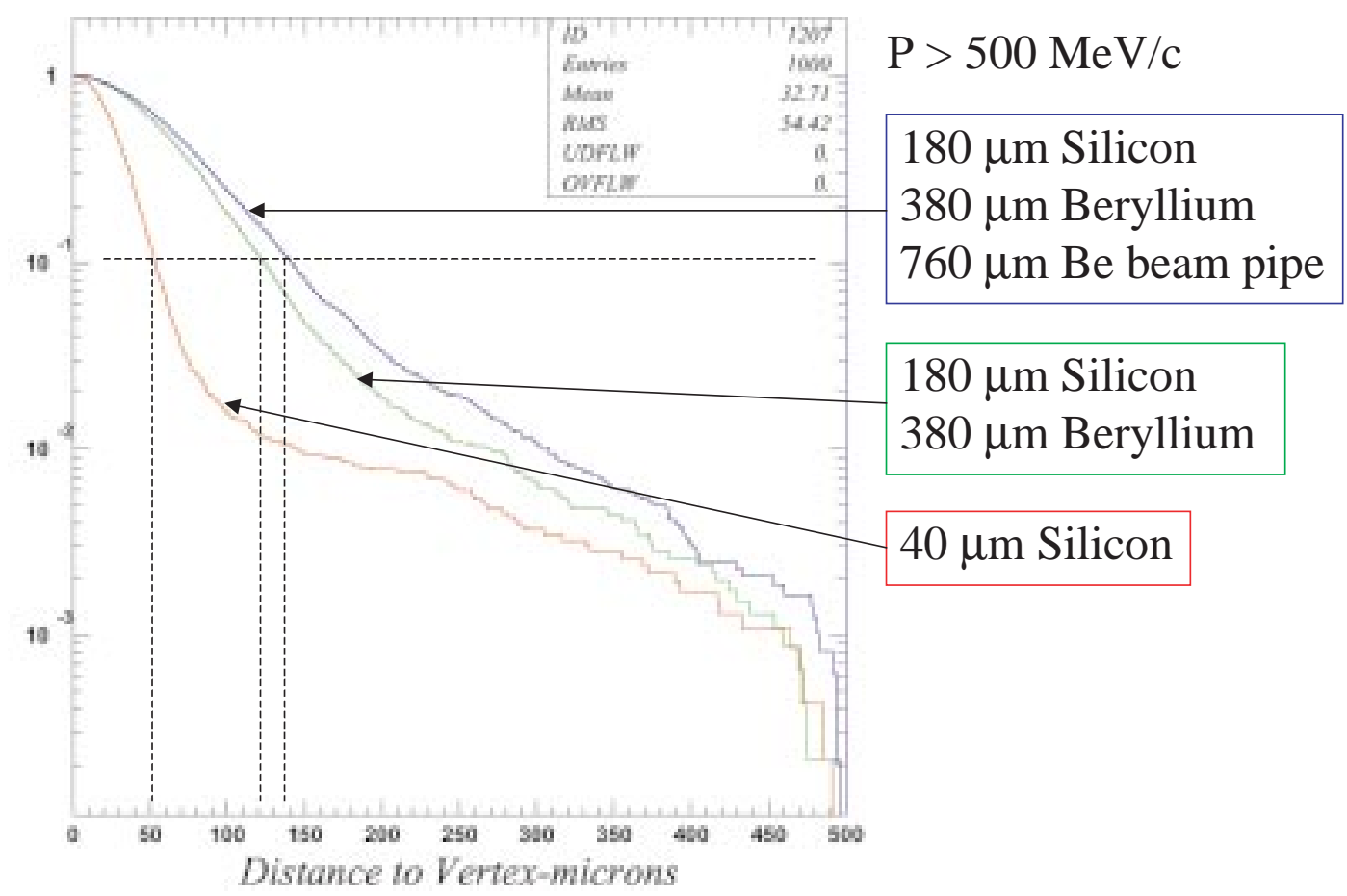

Fig. 2. Fraction of primary tracks that do not get excluded as a function of impact parameter cut. Shows the ability to isolate primary tracks from the D vertex.

We have parameterized the essential features of CCD and APS technologies. Using resolutions attained in the SLD vertex detector, reconstruction values found from STAR simulations, and detector thickness achieved by other groups, our preliminary analysis demonstrates that it is possible to reconstruct $\mathrm{D}$ mesons in STAR using the $\pi \mathrm{K}$ channel. Some parameters of this analysis are one D per event, a $0.76 \mathrm{~mm}$ beryllium beam pipe at $2 \mathrm{~cm}$ radius, $40 \mu \mathrm{m}$ thick silicon first layer at $2.8 \mathrm{~cm}$ radius and a vertex distance of closest ap- 
proach cut of $150 \mu \mathrm{m}$. In this scenario 120 days of RHIC running at STAR will produce a signal to error of 3 . This analysis is quite preliminary. Corrections for less than $100 \%$ tracking efficiency in the installed silicon tracking chambers will make the situation worse, but optimizing cuts will improve D reconstruction statistics.

\section{Design Considerations}

To more precisely measure the impact parameters of the tracks, the new inner vertex detector will extend the tracking from the SVT vertex detector into a smaller radius. The design being considered would require replacing the presently installed $4 \mathrm{~cm}$ radius beryllium beam pipe with a pipe of $2 \mathrm{~cm}$ or less radius. According to RHIC accelerator specialists this should be possible once the collider operation is well understood, since the beam diameters are small, around a millimeter. The new vertex detector would be two cylinders constructed of ladders placed between the first SVT vertex detector layer at $6 \mathrm{~cm}$ and the beam pipe as shown in the sketch (Figure 3). This layout is considered mechanically feasible since it is similar to the successful VXD3 construction. For STAR it would be desirable to have a much longer detector, because of the large crossing diamond at the RHIC collider. However, increasing the length significantly would be a considerable technical challenge. Most of the physics programs at STAR are not luminosity limited, so we can compromise and limit event selection to the center of the detector. A detector design based on CCDs like VXD3 raises two issues: readout speed and radiation hardness. The expected radiation level in STAR at full design luminosity is expected to be $2 \mathrm{kRad} /$ year which is marginally acceptable although the low energy neutron level is not known and could possibly be an issue. The readout speed is of more concern. The VXD3 readout time is $200 \mathrm{~ms}$ which would not work in the STAR environment because event pileup would make the hit density too high for unambiguous tracking. Designing faster readouts for CCDs, however, is an active field of development and $20 \mathrm{~ms}$ might not be an unreasonable expectation for a 10 million pixel CCD. At this speed multiple events would still pileup during readout, but the hit density would be $32 \mathrm{hits} / \mathrm{cm}^{2}$ which is just tolerable from the standpoint of tracking confusion. The difficulty in tracking comes from projecting the tracks from outside in. As long as the hit density on the surface of the inner vertex detector is not large compared to the track projected cone of uncertainty the accidental association with hits from wrong events is a limited problem. The pixel occupancy in this case would be $0.1 \%$ if 9 pixels are populated for each hit. The appeal of CCDs for this application is our very stringent requirements on multiple scattering. CCDs can be thinned and mounted with a minimum of support material. They can be cooled with gas flow and developments are underway [3] to mount them 
under tension thus avoiding any support backing.

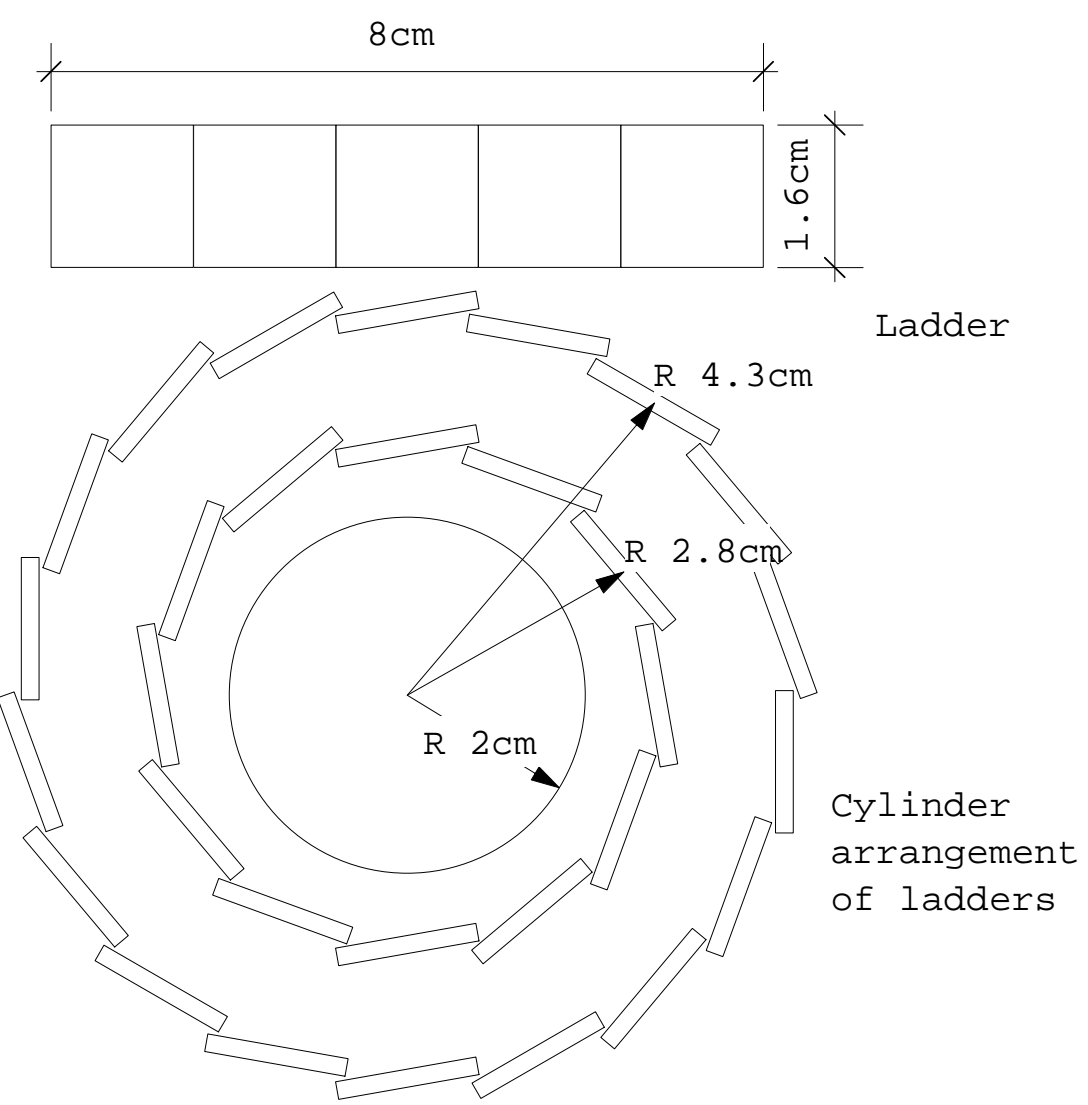

Fig. 3. Vertex detector geometry with ladders of chips arranged in two concentric cylinders around the beam pipe.

A detector design based on the alternate CMOS APS technology potentially overcomes the CCD's limiting factors, poor radiation hardness and readout speed. The CCDs are vulnerable to radiation induced trapping sites because the charge must be transported several centimeters through the silicon. This is not an issue for the APS and it should be as radiation hard as current small geometry CMOS devices. By taking advantage of the ability to design CMOS electronics into the detector chip we hope to achieve readout speeds of less than $2 \mathrm{~ms}$. This is not an unreasonable expectation, since video CMOS APS chips have been built which can be read out at a rate of 1 giga pixel/sec[7]. With a 2 ms readout time we have a small probability for pileup, so the maximum hit density on the detector is set by the track multiplicity of a single central collision. This hit density is 11 hits $/ \mathrm{cm}^{2}$ which given 9 pixels per hit, means an occupancy of $0.04 \%$ for a 20 micron square pixel device.

The APS clearly has potential advantages, but this technology is young and has yet to be used as a particle detector in a working experiment. There are a number of technical issues to be addressed before these devices are ready to be included in an experiment. We will develop a readout scheme and mechan- 
ical approach that will allow us to use these devices in a STAR inner vertex detector. In the interest of minimizing mass we are exploring a mechanical design in which 5 thinned APS chips are joined in a ladder structure that can be supported by tension at the ends of the ladder. A monolithic structure like that used for CCDs is not feasible because the present CMOS dies are limited to $2 \mathrm{~cm}$ on a side. Dies can be joined on a wafer, but this requires special processing and raises yield concerns. As shown in Figure 4 we will attempt a chip design that puts the bulk of the readout and support functions on one side of the chip so that a ladder can be formed with a minimum of dead space between chips. The planned readout scheme takes advantage of the sparse pixel filling and will limit the off chip data transfer requirements. Having the detector in CMOS allows us to design data sparsification right on the detector chip. The sparsification scheme illustrated in Figure 4 checks row by row, skipping empty rows, and then checks column by column for occupied pixels in the row. We hope in this design to have a limited number of connections to the chip. We would have common data and address busses along the inactive edge of the detector chip that would be wire bound jumpered chip to chip. This approach minimizes connections and keeps the dead area on the side of the ladder where it can be covered by slightly overlapping ladders. With data sparsification it is hoped that all the data in an event can be transferred in

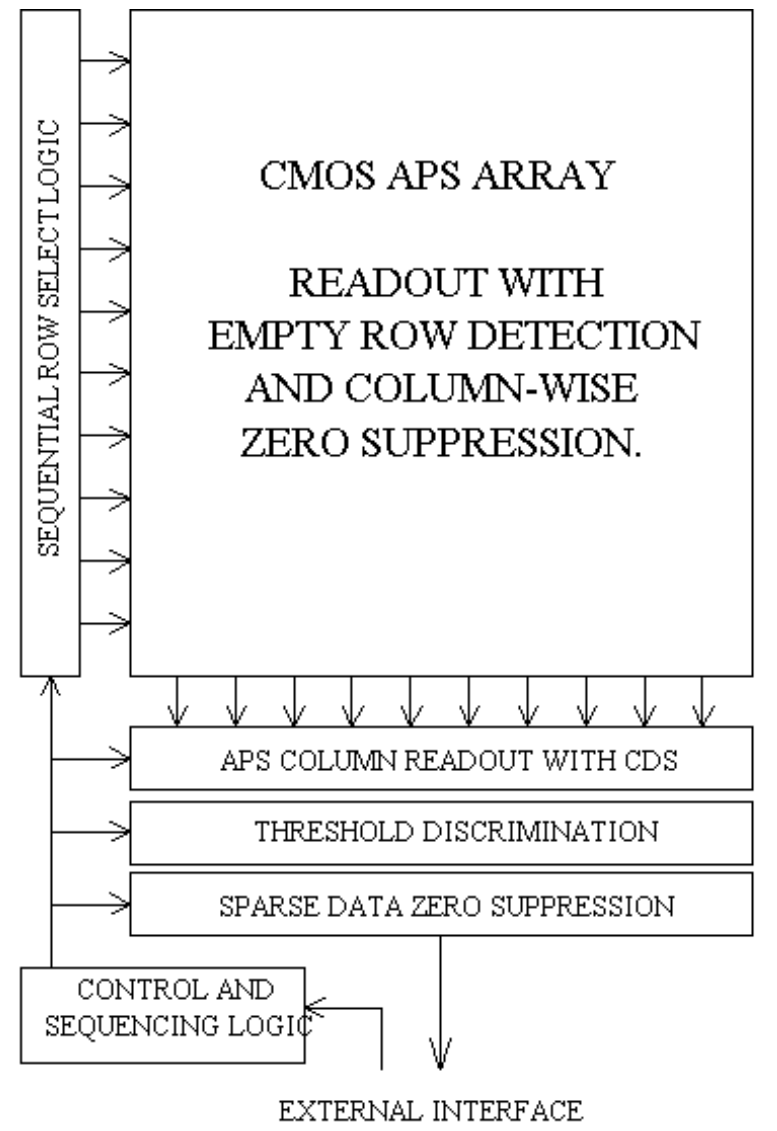

Fig. 4. APS with sparsification on one edge. 
under $2 \mathrm{~ms}$ without a large power penalty. The idea is to limit water cooling to support electronics at the end of the ladders and rely on chilled air alone for cooling the main part of the ladder surface.

\section{Conclusion}

Due to high track density and low particle momentum, detecting D mesons at STAR pushes current vertex detector technology. CCDs may be capable of the task, but the CMOS APS technology shows the best match for the conditions at RHIC.

\section{References}

[1] D. Lynn et al, The STAR silicon vertex tracker: a large area silicon drift detector NIM A 447 (2000) 264.

[2] K. Ave et al, Design and performance of the SLD vertex detector: a 307 Mpixel tracking system, NIM A 400 (1997) 287.

[3] A. Gillman, The CCD Option for LC This conference, Vertex 2000 (2000).

[4] The ALICE collaboration, ALICE Technical Design Report of the Inner Tracking System (ITS), CERN/LHCC 99-12, ALICE TDR 4, 18 June 1999.

[5] J.D. Berst et al, A Monolithic Active Pixel Sensor for Charged Particle Tracking and Imaging using Standard VLSI CMOS Technology, http://wwwlepsi.in2p3.fr , LEPSI-99-15.

[6] M. Winter, Monolithic Motivation for an Inner DetectorActive Pixel CMOS for $L C$, This conference, Vertex 2000 (2000)

[7] S. Kleinfelder, S. Lim, X. Liu, A.E. Gamal, A 10,000 Frames/s 0.18 $\mu \mathrm{m}$ CMOS Digital Pixel Sensor with Pixel-Level Memory LC, Submitted to ISSCC 2001. 